

\title{
JOSHA`S CRITICAL REVIEW OF „PEMBROLIZUMAB PLUS CHEMOTHERAPY IN METASTATIC NON-SMALL-CELL LUNG CANCER”.
}

\author{
By L. Gandhi et al. N Engl J Med, April 16, 2018, DOI: 10.1056/NEJMoa1801005
}

In this double-blind, phase 3 trial, 616 patients with metastatic nonsquamous NSCLC without sensitizing EGFR or ALK mutations were randomly assigned to receive pemetrexed and a platinum-based drug plus either $200 \mathrm{mg}$ of pembrolizumab, a monoclonal antibody against programmed death (PD-1), or placebo every 3 weeks for 4 cycles, followed by up to a total of 35 cycles of either combination as maintenance therapy. After a median follow-up of 10.5 months, the estimated rate of overall survival at 12 months was $69.2 \%$ in the pembrolizumab-combination group versus $49.4 \%$ in the placebo combination group $(\mathrm{P}<0.001)$. Median progression-free survival was 8.8 months $(95 \% \mathrm{Cl}, 7.6$ to 9.2$)$ in the pembrolizumab-combination group and 4.9 months in the placebo-combination group $(\mathrm{P}<0.001)$. Adverse events of grade 3 or higher occurred in $67.2 \%$ of the patients in the pembrolizumab-combination group and in $65.8 \%$ of those in the placebo-combination group.

JOSHA's Conclusion: SIGNIFICANT PROGRESS WITHOUT ADDITIONAL GRADE 3 TOXICITY

A striking benefit in overall survival was observed, and a less striking benefit in progression-free survival. This is quite in contrast to other recent studies with checkpoint inhibitors demonstrating a significant event-free survival benefit but did not demonstrate an overall survival benefit - as yet. Perhaps disease control without added toxicity is a better strategy than trying to eradicate the last cancer cells with added treatment toxicity? The innovative therapeutic strategies based on concepts of clonal evolution proposed by Cassandra Willyard (Cancer: An evolving threat, Nature 532, 166, 2016) could be of relevance here. Also, the role of type and duration of maintenance therapy should be studied further. 
PD-1 blockers are approved for several different types of malignancies. It is assumed that their efficacy is based on an accrual of CD8-psitive T cells into the tumors with suitable immune gene patterns and the interaction of tumor cells with $T$ cells and antigenpresenting cells. In this context it is interesting to note that adjuvant $200 \mathrm{mg}$ pembrolizumab every 3 weeks for up to 1 year resulted in significantly longer recurrencefree survival than placebo in a phase III trial with $>1000$ patients who had resected cutaneous melanoma with metastasis to regional lymph nodes, regardless of PD-1 ligand expression of melanoma-positive lymph nodes at enrollment. New toxicities were not observed. About $15 \%$ of the pembrolizumab-treated patients experienced grade 3 to 5 toxicities during the 1-year-treatment, which seems to be meaningfully less toxic than the profile of the pricey anti-CTL4 antibody ipilimumab. The trial (EORTC 1325) is planned to continue to its secondary endpoints, including overall survival (A.M.M.Eggermont et al, N Engl J Med 2018;378:1789-801. DOI: 10.1056/NEJMoa1802357).

\section{Original Article}

\section{Pembrolizumab plus Chemotherapy in Metastatic Non-Small-Cell Lung Cancer}

N Engl J Med

April 16, 2018

DOI: 10.1056/NEJMoa1801005

\section{Authors}

L. Gandhi, D. Rodríguez-Abreu, S. Gadgeel, E. Esteban, E. Felip, F. De Angelis, M. Domine, P. Clingan, M.J. Hochmair, S.F. Powell, S.Y.-S. Cheng, H.G. Bischoff, N. Peled, F. Grossi, R.R. Jennens, M. Reck, R. Hui, E.B. Garon, M. Boyer, B. Rubio-Viqueira, S. Novello, T. Kurata, J.E. Gray, J. Vida, Z. Wei, J. Yang, H. Raftopoulos, M.C. Pietanza, and M.C. Garassino, for the KEYNOTE-189 Investigators*

\section{Original Abstract}


Background First-line therapy for advanced non-small-cell lung cancer (NSCLC) that lacks targetable mutations is platinum-based chemotherapy. Among patients with a tumor proportion score for programmed death ligand 1 (PD-L1) of $50 \%$ or greater, pembrolizumab has replaced cytotoxic chemotherapy as the first-line treatment of choice. The addition of pembrolizumab to chemotherapy resulted in significantly higher rates of response and longer progression-free survival than chemotherapy alone in a phase 2 trial.

Methods In this double-blind, phase 3 trial, we randomly assigned (in a 2:1 ratio) 616 patients with metastatic nonsquamous NSCLC without sensitizing EGFR or ALK mutations who had received no previous treatment for metastatic disease to receive pemetrexed and a platinum-based drug plus either $200 \mathrm{mg}$ of pembrolizumab or placebo every 3 weeks for 4 cycles, followed by pembrolizumab or placebo for up to a total of 35 cycles plus pemetrexed maintenance therapy. Crossover to pembrolizumab monotherapy was permitted among the patients in the placebo-combination group who had verified disease progression. The primary end points were overall survival and progression-free survival, as assessed by blinded, independent central radiologic review.

Results After a median follow-up of 10.5 months, the estimated rate of overall survival at 12 months was $69.2 \%$ (95\% confidence interval [Cl], 64.1 to 73.8 ) in the pembrolizumabcombination group versus $49.4 \%(95 \% \mathrm{Cl}, 42.1$ to 56.2$)$ in the placebocombination group (hazard ratio for death, $0.49 ; 95 \% \mathrm{Cl}, 0.38$ to $0.64 ; \mathrm{P}<0.001$ ). Improvement in overall survival was seen across all PD-L1 categories that were evaluated. Median progressionfree survival was 8.8 months $(95 \% \mathrm{Cl}, 7.6$ to 9.2$)$ in the pembrolizumab-combination group and 4.9 months $(95 \% \mathrm{Cl}, 4.7$ to 5.5$)$ in the placebo-combination group (hazard ratio for disease progression or death, $0.52 ; 95 \% \mathrm{Cl}, 0.43$ to $0.64 ; \mathrm{P}<0.001)$. Adverse events of grade 3 or higher occurred in $67.2 \%$ of the patients in the pembrolizumab-combination group and in $65.8 \%$ of those in the placebo-combination group.

\section{Conclusions}


In patients with previously untreated metastatic nonsquamous NSCLC without EGFR or ALK mutations, the addition of pembrolizumab to standard chemotherapy of pemetrexed and a platinum-based drug resulted in significantly longer overall survival and progression-free survival than chemotherapy alone. (Funded by Merck; KEYNOTE-189 ClinicalTrials.gov number, NCT02578680.)

\section{Author Affiliations}

From NYU Perlmutter Cancer Center, New York (L.G.); Complejo Hospitalario Universitario Insular Materno-Infantil de Gran Canaria, Universidad de Las Palmas de Gran Canaria, Las Palmas de Gran Canaria (D.R.-A.), Hospital Universitario Central de Asturias, Oviedo (E.E.), Vall d'Hebron University, Vall d'Hebron Institute of Oncology, Barcelona (E.F.), and Fundación Jiménez Díaz (M.D.) and Hospital Universitario Quirón Madrid (B.R.-V.), Madrid - all in Spain; Karmanos Cancer Institute, Detroit (S.G.); Integrated Health and Social Services Centres, Montérégie Centre, Greenfield Park, QC (F.D.A.), and Sunnybrook Health Sciences Centre, Toronto (S.Y.-S.C.) - both in Canada; Southern Medical Day Care Centre, Wollongong, NSW (P.C.), Epworth Healthcare, Richmond, VIC (R.R.J.), Westmead Hospital and University of Sydney, Sydney (R.H.), and Chris O'Brien Lifehouse, Camperdown, NSW (M.B.) - all in Australia; Otto Wagner Hospital, Vienna (M.J.H.); Sanford Health, Sioux Falls, SD (S.F.P.); Thoraxklinik, Heidelberg (H.G.B.), and LungenClinic, Airway Research Center North, German Center for Lung Research, Grosshansdorf (M.R.) - both in Germany; Davidoff Cancer Center, Tel Aviv University, Petah Tikva, Israel (N.P.); Ospedale Policlinico San Martino, Genoa (F.G.), University of Turin, Azienda Ospedaliero-Universitaria San Luigi, Orbassano (S.N.), and Fondazione IRCCS Istituto Nazionale dei Tumori, Milan (M.C.G.) - all in Italy; David Geffen School of Medicine at UCLA, Los Angeles (E.B.G.); Kansai Medical University Hospital, Osaka, Japan (T.K.); Moffitt Cancer Center, Tampa, FL (J.E.G.); and Merck, Kenilworth, NJ (J.V., Z.W., J.Y., H.R., M.C.P.).

JOSHA'S Critical Reviews in Oncology and related areas of medicine and science will focus on recently published clinical and non-clinical studies and discoveries. Our editors 
feel that there is a strong need for expert opinions on studies and discoveries that may potentially impact on patient care. While any judgment will, of course, be a personal and subjective opinion of our editors with all its limitations, we do hope this service will be helpful for our readers and stimulate thoughts and reflections in face of ever more rapid introductions of new drugs and therapeutic strategies in these days. We do encourage comments by our readers! 